\title{
EXAMINANDO LA TEORÍA VERIFICACIONISTA DEL SIGNIFICADO
}

\section{AranXa Pizarro}

Pontificia Universidad Católica del Perú

Resumen: El presente trabajo busca analizar la teoría verificacionista del significado propuesta por el positivismo lógico. De acuerdo a esta teoría, solo los enunciados verificables por medio de la observación empírica tienen significado. Nuestro propósito es mostrar las razones por las cuales la teoría verificacionista del significado colapsa. Para ello, examinaremos tanto las críticas internas como externas. En las críticas internas mostraremos los intentos fallidos de los positivistas lógicos en formular un criterio de verificación débil adecuado. En las críticas externas nos centraremos en las formuladas por J.L. Austin a partir de su teoría de los enunciados realizativos.

Palabras claves: significado, verificación, positivismo lógico, Austin, enunciados "realizativos"

Abstract: This paper purports to analyze the verificationist theory of meaning proposed by logical positivism. According to this theory, only sentences verifiably by means of empirical observation have meanings. Our purpose is to show the reasons why the verificationist theory collapses. In order to do so, we will examine both the internal and external critiques to it. Among the internal critiques, we will show the logical positivists' failed attempts to formulate an adequate weak verification criterion. Among the external critiques, we will focus on the ones formulated by J.L. Austin on the basis of his theory of performative utterances.

Key words: meaning, verification, logical positivism, J.L. Austin, "performative" utterances 


\begin{abstract}
"En cuanto el hombre se sirve del lenguaje para establecer una relación viva consigo mismo o con sus semejantes, el lenguaje no es ya un instrumento, no es ya un medio, es una manifestación, una revelación del ser íntimo y del vínculo psíquico que nos une al mundo y a nuestros semejantes".

M. Mearleau- Ponty, Fenomenología de la percepción ${ }^{209}$
\end{abstract}

\title{
§ 1. Introducción
}

El presente trabajo busca exponer algunos motivos por los cuales la teoría verificacionista del significado defendida por el positivismo lógico fracasó. Para lograr esto, en la primera sección vamos a definir qué significa y qué plantea la teoría verificacionista del significado. Al hacerlo, vamos a analizar la diferencia entre el carácter fuerte de verificación y el carácter débil de verificación. Con la ayuda de ejemplos, buscaremos no solo aclararlos, sino también mostrar sus puntos débiles. En la segunda sección nos centraremos en los problemas que enfrenta esta teoría. Con el fin de lograr una mejor comprensión de las críticas que enfrentó la teoría verificacionista del positivismo lógico, se separará a la sección en dos: la primera tratará específicamente sobre las críticas internas de los mismos positivistas lógicos a su teoría y la segunda se centrará en la crítica externa de J.L. Austin a partir de los enunciados realizativos. En esta sección se responderá directamente a la pregunta que motivó, desde sus inicios, a este trabajo: ¿Por qué fracasa la teoría del significado del positivismo lógico? Finalmente, se establecerá claramente qué puede concluirse a partir de lo dicho a lo largo de todo el trabajo.

\section{§ 2. Teoría verificacionista del significado}

El movimiento filosófico conocido como positivismo lógico (también conocido como empirismo lógico o cientificismo empírico $\left.{ }^{210}\right)$, se originó alrededor de 1920

209 Merleau-Ponty, Maurice, Fenomenología de la Percepción, México D.F.: Fondo de Cultura Económica, 1957, p. 213.

210 Cf. MacLennan, Bruce, From Pythagoras to the Digital Computer: The Intellectual Roots of Symbolic Artificial Intelligence, I v., 2009, disponible en: http://web.eecs.utk.edu/ mclennan/ 
en Austria con la formación del Círculo de Viena. Las doctrinas filosóficas del positivismo lógico se gestaron a partir de la lectura tanto de la obra de Bertrand Russell, como del Tractatus Logico-Philosophicus de Ludwig Wittgenstein ${ }^{21 !}$. Además de la enorme influencia de estos autores, los positivistas lógicos se autoproclamaban herederos del empirismo de Locke, Berkeley y Hume.

Una de las metas más importantes para los positivistas lógicos fue demostrar que la metafísica era puro sinsentido y, así, rechazarla por completo ${ }^{212}$. Ahora bien, este rechazo debía basarse en argumentos claros y sólidos, para no caer en puro criticismo infundado ${ }^{213}$. Para lograr esto, los positivistas lógicos establecieron que todo nuestro conocimiento del mundo debía estar basado en la experiencia sensible; por lo cual, toda oración cuya verdad no pueda ser verificada por la experiencia debe considerarse, no solo injustificada, sino un sinsentido ${ }^{214}$ (como la metafísica, la ética y la teología). Esto es lo que los positivistas lógicos llamaron el principio de verificación.

Además del principio de verificación, los positivistas lógicos desarrollaron una comprensión particular de la naturaleza de los problemas filosóficos a partir de su lectura del Tractatus. Según esta lectura, los problemas filosóficos no son más que meras confusiones del lenguaje ${ }^{215}$. Así, la labor principal de la filosofía era clarificar lógicamente los pensamientos ${ }^{216}$ expresados por medio del lenguaje. Es a partir de esta lectura del Tractatus, que los positivistas lógicos creen lo siguiente: (i) la filosofía se encarga del análisis lingüístico, (ii) el análisis lingüístico es fundamentalmente análisis del significado y de la forma lógica, (iii) para determinar la naturaleza del significado se debe establecer las condiciones

2 II Cf. Ayer, Alfred J., Language, truth and logic, London: Penguin Books, 1936, p. 9.

212 Cf. Speaks, Jeff, 20II, “Verificationism”, en: Separata del curso Philosophy 83104: Proseminar of the University of Notre Dame, disponible en: http://www3.nd.edu/ jspeaks/ courses/20II-I2/83I04/handouts/verificationism.pdf

213 Cf. Ayer, Alfred J., op. cit., p. 9.

214 Cf. Speaks, Jeff, op. cit.

215 "El libro trata los problemas filosóficos y muestra -según creo- que el planteamiento de estos problemas descansa en la incomprensión de la lógica de nuestro lenguaje" (Wittgenstein, Ludwig, Tractatus Logico-Philosophicus, traducción de Jacobo Muñoz e Isidoro Reguera, Madrid: Alianza Editorial, 2012, p. 55).

216 "El objetivo de la filosofía es la clarificación lógica de los pensamientos. La filosofía no es una doctrina, sino una actividad. Una obra filosófica consta esencialmente de aclaraciones. El resultado de la filosofía no son $<<$ proposiciones filosóficas $>>$, sino el que las proposiciones lleguen a clarificarse. La filosofía debe clarificar y delimitar nítidamente los pensamientos, que de otro modo son, por así decirlo, turbios y borrosos" (ibid., 4.II2, pp. 82-83). 
de verdad de las oraciones. Solo haciendo esto los problemas filosóficos serían disueltos. Esta aproximación al significado es lo que se conoce como la teoría verificacionista del significado, cuya tesis fundamental es la siguiente:

(TI) El significado de una oración es su método de verificación ${ }^{217}$

Es importante que se observe que para los positivistas lógicos solo las oraciones declarativas expresan proposiciones, es decir, solo aquellas oraciones pueden ser verdaderas o falsas. De esta manera, las proposiciones son el significado de una oración declarativa y, por lo tanto, solo ellas pueden ser sujetas a verificación. Así, para los positivistas lógicos, una oración significa algo si y solo si es posible verificar su valor de verdad, es decir, si es posible verificar si es verdadera o falsa. Por lo cual, no toda oración significativa tiene que ser verdadera: puede haber oraciones que tengan significado pero que sean falsas.

Comparemos, por ejemplo, las siguientes oraciones:

(I) El gato de Mariana es negro

(2) La sustancia es densa

Mientras que podemos verificar el valor de verdad de la oración (I) apelando a nuestra capacidad perceptiva, el valor de verdad de la oración (2) no puede ser verificado por medio de la observación empírica. Así, para los positivistas lógicos, la oración (I) sí tendría significado mientras que la oración (2) sería un sinsentido. Cabe señalar que lo fundamental aquí no es que la verificación haya ya sido establecida, sino que pueda establecerse. En otras palabras, el criterio de significación para los positivistas lógicos no era la verificación actual, sino la verificación posible. Ahora surge la pregunta: ¿En qué consiste el método de verificación? Para los positivistas lógicos, el método de verificación era el método de observación de las ciencias empíricas y no cualquier tipo de verificación personal, religiosa, etc. Entonces:

217 "Decimos que una oración es factualmente significativa para un sujeto determinado, si, y sólo si, él sabe cómo verificar la proposición que ella se propone expresar - es decir, si sabe qué observaciones lo llevarían, bajo ciertas condiciones, a aceptar la proposición como verdadera, o a rechazarla como falsa" (Ayer, Alfred J., op. cit., p.16. La traducción es de la autora del artículo). 
(T2) El método de verificación es la observación ${ }^{218}$

Debemos aclarar que los positivistas lógicos solo admiten dos tipos de conocimiento: el de las proposiciones analíticas de las matemáticas y la lógica, y el de las proposiciones sintéticas que son siempre empíricas. La teoría del significado de los positivistas lógicos versa solamente sobre las proposiciones sintéticas; en ningún momento cuestiona a las proposiciones analíticas porque, por su propia naturaleza tautológica, ellas son verdaderas en todos los mundos posibles. En otras palabras, ellas son verdaderas independientemente de cómo sea el mundo en el cual se evalúan. Esto quiere decir que su valor de verdad no es verificable por medio de la experiencia empírica y, por lo tanto, no aportan ningún conocimiento sobre el mundo.

Ahora, cuando los positivistas hablan de la observación no se están refiriendo a cualquier tipo de observación. Como ya se mencionó en los párrafos anteriores, ellos se están refiriendo a la observación tal como es entendida en las ciencias empíricas. Según esta concepción, la observación tiene que satisfacer ciertos requisitos: (i) teóricamente guiada y (ii) repetible en condiciones diversas. De acuerdo con el primer requisito, la observación presupone no solo un conocimiento previo, sino también haber desarrollado una destreza particular que le permite al científico ver cosas en la naturaleza que otras personas no pueden ver. Por ejemplo: si un médico y yo vemos mi radiografía, a pesar de que ambos tengamos en frente la misma imagen, para él una sombra puede ser un tumor mientras que para mí puede pasar desapercibida. Según el segundo requisito, no basta con que el científico haya observado cierto fenómeno una sola vez. Para que la experiencia particular del científico cuente como una observación científica tiene que poder realizarse múltiples veces en distintas circunstancias y por distintos observadores adecuadamente entrenados.

Los positivistas lógicos distinguen entre dos criterios de verificación: la verificación práctica y la verificación en principio ${ }^{219}$. Debe agregarse que, debido a que hay ciertas divergencias entre los mismos positivistas lógicos con respecto a este tema, lo dicho a continuación se basa en la distinción que A.J. Ayer presenta en la primera edición de su libro Lenguaje, verdad y lógica. Retomando lo 
dicho previamente, la mayoría de nosotros tiende a aceptar como verdaderas muchas proposiciones que no han sido todavía verificadas por la experiencia sensible. Sin embargo, a pesar de que muchas de estas proposiciones sí podrían ser verificadas si decidiéramos hacerlo, hay otras proposiciones que no podrían ser verificadas todavía. Un ejemplo de una proposición que todavía no puede ser verificada es la que expresa la siguiente oración:

(3) Hay vida en Júpiter

Es cierto que no poseemos las herramientas necesarias para verificar la verdad o falsedad de esta proposición. Sin embargo, esto no quiere decir que no pueda ser verificada más adelante ${ }^{220}$. De esta manera, es una proposición que puede ser verificada en principio más no en la práctica y, por lo tanto, la oración (3) es significativa.

Otra distinción importante para los positivistas lógicos a la hora de establecer un criterio claro que defina su método de verificación es el siguiente: hay verificaciones de carácter fuerte y de carácter débil. Una proposición es verificable, en el sentido fuerte, si y solo si su verdad puede ser establecida de manera concluyente por la experiencia sensible. La verificación de carácter fuerte, por su propia naturaleza concluyente, en caso de ser asumida, sería insostenible como criterio de significación porque haría de las leyes de la naturaleza simples sinsentidos. Por ejemplo, consideremos la oración:

Todos los hombres son mortales

Si se adoptara un principio de verificación fuerte, esta oración carecería de significado ya que para verificarla deberían haber muerto todos los hombres antes de poder determinar si esta proposición es verdadera o falsa ${ }^{221}$. Entonces, ise la debería considerar como sinsentido? ${ }^{222}$ No. Se sabe que todos los seres humanos son mortales, no porque se pueda inferir deductivamente la verdad de esta proposición, sino porque hay suficientes casos, por el momento, que justifican inductivamente la proposición de que todos los humanos son mor- 
tales. Puede que, en el futuro, se dé una anomalía pero hasta que eso suceda se sigue afirmando que todos los seres humanos son mortales.

Dado que casi todas las proposiciones generales basadas en la observación científica tienen una justificación inductiva (probable) y no deductiva (concluyente), la verificación fuerte como criterio de significación es inadecuada y debe ser descartada. Los positivistas lógicos se dan cuenta de que necesitan un criterio de verificación débil. La tarea de formular este criterio fue mucho más difícil de lo que anticiparon y finalmente fue una de las causas por las cuales colapsó la teoría verificacionista del significado:

"En cuanto a la validez del principio de verificación en la forma en que lo hemos enunciado, daremos una demostración en este libro. Se demostrará, en efecto, que todas las proposiciones que tienen contenido fáctico son hipótesis empíricas, y que la función de una hipótesis empírica es la de proporcionar una regla para anticipar la experiencia. Ello significa que toda hipótesis empírica debe referirse a alguna experiencia real o posible, de modo que, un enunciado sin relación con experiencia alguna no es una hipótesis empírica y, en consecuencia, carece de contenido fáctico. Pero esto es precisamente lo que afirma el principio de verificabilidad"223.

A modo de conclusión, debe quedar claro que los positivistas lógicos sostienen lo siguiente: (i) toda proposición que versa sobre el mundo tiene un contenido fáctico, (ii) toda proposición fáctica tiene la forma de hipótesis empírica, (iii) toda hipótesis empírica puede ser verificada a partir de la experiencia sensible, (iv) solo las oraciones que expresan proposiciones que pueden ser verificadas tienen significado ( $v$ ) solo las oraciones declarativas expresan proposiciones fácticas. Por lo tanto, solo las oraciones declarativas tienen significado. De esta manera, todas las demás oraciones son calificadas como sinsentidos. Los positivistas lógicos sí reconocen en los enunciados éticos y en los enunciados estéticos la expresión de una emoción ${ }^{224}$. Así, aunque siguen siendo sinsentidos, se les puede entender como enunciados emotivos.

223 Ayer, Alfred J., Lenguaje, verdad y lógica, Buenos Aires: Editorial Universitaria de Buenos Aires, 1965, p. 23.

224 "Algunos positivistas lógicos pensaban que los juicios éticos y estéticos no tenían más significado que eyaculaciones emocionales" (MacLennan, Bruce, op. cit.). 


\section{§ 3. Problemas para la teoría verificacionista del significado}

Alrededor de 1930 el positivismo lógico comenzó a fragmentarse. Este fue el comienzo del fin; aunque algunos miembros del movimiento lo mantuvieron durante las décadas siguientes, puede decirse que terminó con la misma fuerza con la que surgió. Las causas por las que el positivismo lógico colapsó fueron tanto internas como externas. A continuación, se expondrá primero la gran crítica interna. Luego, se retomará la crítica externa a partir de J.L. Austin.

\section{$\S$ 3.I. Críticas internas}

Como ya se mostró en la sección anterior, el carácter fuerte de verificación resultó muy rígido y, por lo tanto, inadecuado. Tan pronto como los positivistas lógicos se dieron cuenta de esto, se dedicaron a buscar una formulación débil del principio de verificación que funcione como criterio necesario para determinar el significado de una oración declarativa. Sin embargo, una de las razones por las que el positivismo lógico colapsó fue porque nunca lograron formular de manera clara en qué consistía el principio débil de verificación. Esto es precisamente lo que se va a exponer en esta sección del trabajo. A continuación, la primera formulación de $A y e r^{225}$ en torno al carácter débil de la verificación:

"En consecuencia, volvemos a caer en el sentido débil de verificación. Decimos que la preguntar que hay que formular a propósito de cualquier presunto enunciado fáctico no es "ipodrá una observación establecer la verdad o falsedad de ese enunciado en forma lógicamente cierta?" sino, simplemente, "ihabrá alguna observación que sea pertinente para la determinación de su verdad o falsedad?" Sólo en el caso en que se dé una contestación negativa a esta segunda pregunta concluimos que el enunciado en consideración carece de sentido.

Para aclarar más nuestra posición, hemos de formularla de otro modo. Llamemos proposición experiencial a una proposición que registra una observación real o posible. Podemos decir entonces que lo que distingue a una proposición fáctica genuina no es el hecho de que debería ser equivalente a una proposición experiencial, o a un número finito cualquiera de proposiciones experienciales, sino simplemente que 
de ella, en conjunción con ciertas otras premisas, pueden deducirse algunas proposiciones experienciales que no son deducibles de esas otras premisas por sí solas" 226 .

Lo que hace que una oración empírica tenga significado ya no es que exprese un enunciado que pueda ser constatado como verdadero o falso por algún tipo de observación empírica directa. Ahora, según el carácter débil de verificación, una oración empírica tiene significado si y solo si existe una observación empírica relevante para determinar su verdad o falsedad ${ }^{227}$. ¿A qué se refieren los positivistas lógicos con relevante?

Lo que los positivistas lógicos buscaban con sus criterios de verificación tan rigurosos era imitar los procesos de confirmación y refutación de las hipótesis en las ciencias empíricas. Primero creyeron que el criterio de verificación debía ser la observación empírica directa y concluyente. A esto lo llamaron el carácter fuerte de verificación. Sin embargo, pronto se dieron cuenta de que este criterio era inadecuado porque había oraciones generales como (4) ${ }^{228}$ que serían catalogadas como sinsentidos ya que jamás podrían ser verificadas concluyentemente. De esta manera, lo que buscan con el criterio débil de verificación era rescatar a este tipo de oraciones generales del conjunto de sinsentidos, ya que las ciencias empíricas constantemente hacen uso de ellas (considérese, por ejemplo, oraciones empíricas tales como "Todos los metales se dilatan al ser calentados" o "Todo líquido tiene un punto de ebullición”).

Luego de algunos intentos, los positivistas lógicos creyeron haber encontrado una formulación adecuada del principio de verificación que buscaban. La idea fundamental es la siguiente: una oración tiene significado si y solo si juega un rol indispensable en la derivación de una oración observacional ${ }^{229}$. Por ejemplo, supongamos que queremos saber si la oración "Todos los líquidos tienen un punto de ebullición” tiene significado o no. Según el principio débil de verificación, lo que tendríamos que hacer es intentar derivar lógicamente

226 Ayer, Alfred J., Lenguaje, verdad y lógica, p. 38-39.

227 Cf. Soames, Scott, Philosophical analysis in the twentieth century, New Jersey: Princeton University Press, 2003, p. 282.

228 "Todos los hombres son mortales".

229 Una oración observacional es una oración que sí puede ser constatada mediante la observación empírica. 
una oración observacional a partir de la conjunción entre dicha oración y cualquier otra. Si logramos hacerlo, entonces la oración tendrá significado. Si no lo logramos, será un sinsentido. Claramente, la oración sí tiene significado. Si la conjugamos con la oración "El agua es un líquido", derivaremos lógicamente "El agua tiene un punto de ebullición”. Esta tercera oración puede, en efecto, ser constatada mediante la observación empírica, con lo cual, sí tiene significado (más allá de que sea verdadera o falsa). Por lo tanto, la oración general “Todos los líquidos tienen un punto de ebullición” tiene significado. De esta manera, solo se necesita que la tercera oración observacional tenga alguna relación con la oración general para atribuirle verificabilidad a esta última. Esta relación, como ya se mencionó, no es de manera alguna concluyente, sino solo relevante. Así, al decir relevante, los positivistas lógicos aflojan el vínculo entre el significado de la oración y su método de verificación.

Ahora bien, este carácter débil de verificación era insostenible, a tal punto, que el mismo Ayer se da cuenta y propone un avasallador contraejemplo en la segunda edición del libro Lenguaje, verdad y lógica, cuando lo reformula. En este trabajo vamos a centrarnos solo en la primera definición que Ayer propone porque la segunda, a pesar de que intenta mejorar lo dicho previamente, sigue siendo inexacta y fracasa. El problema fundamental de la primera definición del carácter débil de verificación consiste en que no hay ningún criterio para determinar qué premisa general puede ser utilizada para la inferencia. No solo eso, tampoco hay un criterio que determine qué oración particular puede ser utilizada. Así, al aplicar su principio de manera irrestricta, caen en problemas irresolubles.

Para ejemplificar el gran problema que se acaba de mencionar se usará el siguiente contraejemplo: si todos los unicornios vuelan, entonces mi nombre es Aranxa. Esta vendría a ser la premisa particular, con la cual la premisa general se junta: todos los unicornios vuelan. Así, se concluye por Modus Ponens que mi nombre es Aranxa. Esta es una oración observacional que sí puede ser constatada mediante observación empírica y, por ende, sí tiene significado. Al tener esta oración significado, se le atribuiría (como consecuencia de la aplicación del sentido débil de verificación) significado a la oración general Todos los unicornios vuelan. Este resultado es absurdo para los positivistas lógicos, ya que esta oración debería ser un sinsentido. 
Ahora, si decir que algo tiene significado es decir que puede ser sujeto a verificación, a pesar de que no sea de manera concluyente, icómo podemos decir que esta oración tiene significado, es decir, que puede ser sujeta a verificación si no existen los unicornios? Hay muchos ejemplos similares. Los positivistas lógicos no se dieron cuenta de que pasaron de un criterio de verificación absolutamente rígido al proponer el carácter fuerte, a un criterio de verificación inconsistente con el carácter débil de verificación. Así, los positivistas lógicos nunca pudieron establecer un criterio sólido y claro de observación para verificar las oraciones. Por esta razón y otras más, la teoría verificacionista del significado fue completamente insostenible y colapsó.

Otro problema que vamos a tratar surge cuando, en 1927, se publica el principio de incertidumbre de Heisenberg ${ }^{230}$ de la mecánica cuántica. Este principio establece que la posición y la inercia de una partícula subatómica son siempre inciertas. Es importante que se recalque que este no es un problema epistemológico, es decir, no es que todavía no poseamos los medios para conocer la posición y la inercia de la partícula subatómica. El principio ${ }^{231}$ afirma claramente que las leyes de la física son tales que jamás podremos determinar su posición e inercia exacta porque carecen de ella ${ }^{232}$. Pero, icuál es la relevancia de este descubrimiento para la teoría verificacionista del significado?

Para empezar, se debe recordar la formación científica de la mayoría de los positivistas lógicos y el hecho de que su principio de verificación está inspirado en el método científico. Además, los positivistas lógicos tenían como meta ser absolutamente claros y precisos. La consecuencia directa del principio de incertidumbre era el problema de la medida. No importa cuán sofisticados sean nuestros métodos de medición, el resultado sería siempre solo un aproximado. Lo que sucede es que al medir algo, por ejemplo un árbol, para dar la medida exacta uno tendría que llegar a las partículas más pequeñas de ese árbol. Y, como ya dijimos, estas partículas subatómicas no podrán ser jamás medidas porque carecen de posición. Debido a esto, todas las oraciones que versan sobre la medida de algo en el mundo deberían carecer de significado, es decir, deberían ser consideradas como sinsentidos de acuerdo al criterio

230 Cf. MacLennan, Bruce, op. cit.

231 Según la interpretación de Copenhague.

232 Cf. ibid. 
absolutamente rígido establecido por los positivistas lógicos. Este resultado es, por decir lo menos, extraño, ya que implica que todas nuestras oraciones que versan sobre alguna medida en el mundo serían falsas.

Los positivistas lógicos no toman en consideración los intereses que tenemos en distintas situaciones; es decir, las distintas circunstancias y los distintos contextos nos exigen distintas maneras de aproximarnos a ellas. Por ejemplo, si vas a sacar tu DNI y en el formulario te preguntan cuánto mides, deberías poner un signo de interrogación de acuerdo a los positivistas lógicos ya que para ellos tu medida sería incierta y, por lo tanto, esa pregunta quedaría sin respuesta. Sin embargo, tanto el interés del que pregunta como el interés de que responde no es ni dar ni recibir una medida absolutamente precisa. El interés es simplemente satisfacer un criterio básico y aproximado para poder sacar el DNI. Consideremos otro ejemplo: si un amigo te dice para ir a tomar un café y tú le preguntas dónde queda la cafetería, tu amigo no podría decirte dónde porque este lugar no tendría una posición exacta de la cual hablar y la respuesta sería una incógnita. Pero es evidente que las necesidades diarias del hombre no se suman a la precisión absoluta que requiere la ciencia. De esta manera, al no reconocer que hay distintos contextos donde se requieren distintos niveles de precisión, los positivistas lógicos mutilaron del lenguaje numerosos recursos que resultan útiles y necesarios para comunicarnos.

\section{$\S$ 3.2. Críticas Externas: Austin, la falacia descriptiva y los realizativos}

Como ya hemos mencionado antes, los positivistas lógicos declararon a los enunciados éticos, metafísicos, teológicos y estéticos como sinsentidos. Ellos dicen claramente que estos enunciados, a pesar de tener una forma gramatical correcta ${ }^{233}$, son sinsentidos porque no pueden ser verificados. Así, se establece que los únicos enunciados ${ }^{234}$ que portan conocimiento sobre el mundo y que, por lo tanto, tienen significado son los enunciados declarativos verificables. Austin rechaza radicalmente esta posición. Él se pregunta qué

233 Cf. Austin, J. L., Cómo hacer cosas con palabras, Bacerlona: Paidós, 1982, p. 42.

234 En realidad los enunciados son expresados en oraciones, por eso se habla de oraciones declarativas en la primera parte del trabajo (como explica Austin en la nota al pie de página I de su primera conferencia). 
pasa con las preguntas, con las oraciones valorativas, con las oraciones que expresan deseos, entre otras ${ }^{235}$.

Para Austin, creer que solo tiene significado aquello que describe el mundo es un error. Austin rechaza terminantemente este principio y lo llama la falacia descriptiva. Él cree que los positivistas lógicos han caído ciegamente en este problema y por eso busca la manera de refutar su posición. Es así como Austin descubre los enunciados realizativos. Antes de que se explique qué se entiende por este tipo de enunciados, queremos dar dos ejemplos para mostrar por qué Austin establece que es un error suponer que solo las expresiones que describen el mundo y que, por lo tanto, pueden ser sujetas a verificación, tienen significado. Consideremos los siguientes casos:

Primer ejemplo:

(5) Tú eres alumno de la PUCP

(6) Usted es alumno de la PUCP

Los positivistas lógicos trataban a ambos enunciados como dos oraciones declarativas que podían ser sujetas a verificación. Supongamos que nos dirigimos a Juan y le decimos (5) y (6). Si Juan es un alumno de la PUCP, entonces ambas oraciones son verdaderas, $y$ si Juan no es alumno de la PUCP, entonces ambas oraciones son falsas. Para los positivistas lógicos, ambas oraciones aportan el mismo conocimiento sobre el mundo y, en consecuencia, significan lo mismo. Sin embargo, nótese la diferencia entre tú y usted. Desde el uso cotidiano del lenguaje castellano se sabe que al decir tú se sugiere una relación más cercana con la persona en cuestión. Por otro lado, el usted expresa, en la mayoría de casos, una mayor distancia. Usualmente, utilizamos el usted cuando nos referimos a alguien que requiere una muestra explícita de respeto debido a su jerarquía o autoridad. Esto evidencia cómo, aunque ambas oraciones tengan el mismo valor de verdad, hay una diferencia entre el uso del término tú y el uso del término usted que comunica algo distinto en cada caso, que el criterio de significación de los positivistas lógicos no logra ni contemplar ni capturar. 
Esto muestra que debe diferenciarse con claridad lo siguiente: (i) la manera como se enuncia algo ${ }^{236}$, (ii) de lo que se enuncia, es decir, de lo dicho.

Segundo ejemplo:

(7) Marta tiene mi tijera

(8) Martita tiene mi tijera

El segundo ejemplo propuesto muestra el mismo problema. Al reemplazar Marta por Martita no se está cambiando el valor de verdad de las oraciones: si Marta tiene mi tijera, ambas oraciones son verdaderas y si no la tiene, ambas son falsas. Sin embargo, la segunda oración comunica algo que no comunica la primera oración. El uso del diminutivo transmite cierta cercanía o afecto que la primera oración no transmite. Aquí se muestra claramente la diferencia entre los componentes de la oración y la oración entendida como unidad. Pero, como ya mencionamos previamente, los positivistas lógicos desacreditaron la importancia de estas diferencias. Ellos solo se preocupaban por el conocimiento que aportaba la oración entendida como unidad y, así, descartaban que cualquier componente de dicha oración, en sí mismo, pueda aportar algo nuevo no-descriptivo sobre lo dicho. Austin reconoce esto como un grave error. A continuación se muestra la postura de Austin en torno a lo dicho:

"A menudo, también usamos expresiones en modos que, por lo menos, están más allá del ámbito de la gramática tradicional. Se ha llegado a advertir que muchas palabras especialmente desconcertantes, incluidas en enunciados que parecen ser descriptivos, no sirven para indicar alguna característica adicional, particularmente curiosa o extraña, de la realidad, sino para indicar ( $y$ no para registrar) las circunstancias en que se formula el enunciado o las restricciones a que está sometido, o la manera en que debe ser tomado, etc. Pasar por alto estas probabilidades, tal como antes era común, es cometer la llamada falacia descriptiva"237.

Retomando lo que ya se mencionó al comienzo de esta sección, Austin descubre que hay distintos tipos de enunciados que no expresan proposiciones, 
es decir, que no pueden ser verificados: los realizativos. Los realizativos no describen nada, por lo cual ellos no pueden ser ni verdaderos ni falsos. Estos son el contraejemplo que Austin usa para justificar por qué la teoría verificacionista del significado de los positivistas lógicos era terriblemente limitada $y$, de esta manera, inaceptable. Pero, iqué es un realizativo? Austin llama a los realizativos enunciados lingüísticos que se disfrazan. Según él, todavía ni los gramáticos ni los filósofos han visto claramente por detrás del disfraz. Como ya mencionamos, los realizativos (A) no “'describen o registran' nada, y no son 'verdaderas o falsas'; y (B) el acto de expresar la oración es realizar una acción, o parte de ella, acción que a su vez no sería normalmente descrita como consistente en decir algo"238. Queremos retomar algunos ejemplos del propio Austin para mostrar qué es un realizativo:

(9) Bautizo este barco María Eugenia

(10) Lego este CD a mi hermano

(II) Te apuesto cien soles que mañana va a llover

En los ejemplos previos "parece claro que expresar la oración (por supuesto en las circunstancias adecuadas) no es ni describir ni hacer aquello que se diría que hago al expresarme así, o enunciar que lo estoy haciendo: es hacerlo"239. Por lo cual, el enunciado realizativo, para serlo, debe cumplir con ciertos parámetros. La marca de un realizativo es la siguiente: (i) debe estar en primera persona del singular, de la voz activa, del presente, indicativo, por ejemplo: Perdóname. En el momento que esto se vuelva ella me perdonó deja de ser un realizativo y se vuelve una oración que puede ser constatada, un constatativo. (ii) Están regulados por convenciones sociales, es decir, dependen de la circunstancia correcta. Si algún elemento necesario para la acción no está en su lugar entonces es un acto fallido, por ejemplo: Te bautizo. Si lo dice un sacerdote entonces, al estar dentro de las convenciones sociales, sería aceptado como un realizativo. Pero, si lo dijese yo caminando por la universidad a un amigo cualquiera, entonces no habría una conexión entre lo dicho y lo que esto representa. De esta manera, no sería un realizativo. (iii) Que el hablante sea sincero, por ejemplo: Te quiero. Si la persona que dice esto no lo dice con honestidad, entonces sus palabras no estarían acompañando sus emociones.

238 lbid., p. 45-46.

239 lbid., p. 46. 
De esta manera, la acción no sería válida y este no podría ser considerado como un realizativo. Estos enunciados no tienen condiciones de verdad sino condiciones de felicidad, es decir, condiciones de uso apropiado (o feliz) y de uso inapropiado (o infeliz).

Como ya hemos ido sugiriendo con los enunciados realizativos, Austin amplía el concepto de significado que los positivistas lógicos habían formulado. Ya no es significativo solo un enunciado que pueda ser empíricamente constatado mediante la observación. Así, las oraciones declarativas se convierten solo en un tipo de enunciado significativo pero se reconoce que hay otro tipo de enunciados significativos, como los realizativos en este caso. De este modo, se muestra que los positivistas lógicos cayeron ciegamente en la llamada falacia descriptiva, como Austin la llama, por la pretensión de utilizar el método científico exacto de verificación en su teoría del significado. Este método era sumamente restrictivo y, desafortunadamente para los positivistas lógicos, el lenguaje ordinario no pudo y, jamás podrá, adecuarse a su rigurosidad.

\section{§ 4. Conclusión}

El propósito principal de este ensayo ha sido exponer las razones por las cuales fracasa la teoría verificacionista del positivismo lógico. Para esto, primero se ha analizado el argumento de los positivistas lógicos a favor de la teoría verificacionista del significado y se ha mostrado que el criterio de verificación que exponen para determinar el significado de una oración declarativa es inadecuado. El criterio de verificación fuerte terminó invalidando los enunciados científicos. Luego, intentaron definir el criterio débil de verificación pero fracasaron al no formularlo de manera clara.

Así, no logran tener condiciones necesarias de verificación con lo cual la teoría verificacionista del significado fracasa. Esto es precisamente lo que hemos mostrado en las críticas que surgen internamente por los propios positivas lógicos. Luego, mostramos las críticas que surgen externamente. Como crítica externa expusimos las objeciones de J.L. Austin de acuerdo con las cuales el significado no se agota en la descripción. Los casos que sirven a Austin para ilustrar su posición son los enunciados realizativos. De esta manera, Austin 
muestra aspectos del significado lingüístico que el positivismo lógico fracasa en capturar. Con lo cual, la concepción del lenguaje da un giro. Se deja de ver al lenguaje solo como meros enunciados y se lo comienza a ver como acción.

Quizá los positivistas podrían haber tenido éxito si hubiesen especificado que solo les interesaba el lenguaje científico, pero su grave error fue pensar que absolutamente todas las manifestaciones del lenguaje funcionaban como el lenguaje de la ciencia. La pretensión absolutista del método científico que los positivistas lógicos glorificaron se mostró deficiente al enfrentarse a la riqueza del lenguaje ordinario que no es sino reflejo de la vida misma. Por eso, el problema central de los positivistas lógicos fue entusiasmarse con los criterios de verificación científicos y creer que, a partir de ellos, podrían analizar al lenguaje natural como al lenguaje de las ciencias exactas. 